\title{
PERSISTENCE OF PERIODIC ORBITS IN PERIODICALLY FORCED IMPACT SYSTEMS
}

\author{
Michal FEČKAN* - Michal POSPÍŠIL** \\ (Communicated by Jozef Džurina)
}

\begin{abstract}
This paper is devoted to the study of persistence of forced periodic solutions for impact systems from single periodic solutions of unperturbed impact equations. An example of planar discontinuous ordinary differential equations is given to illustrate the theory.
\end{abstract}

(C) 2014

Mathematical Institute

Slovak Academy of Sciences

\section{Introduction}

Consider a motion of a single particle in one spatial dimension described by the position $x(t)$ and the velocity $\dot{x}(t)$. We suppose that it is moving under a linear spring, it is weakly damped and forced. So its position satisfies the ordinary differential equation

$$
\ddot{x}+\varepsilon \zeta \dot{x}+x=\varepsilon \mu \cos \omega t \quad \text { if } \quad x(t)<\sigma,
$$

where $\varepsilon \zeta$ measures the viscous damping, $\varepsilon \mu$ is the magnitude of forcing, $\varepsilon$ is a small parameter, and we suppose that the motion is free to move in the region $x<\sigma$ for $\sigma>0$, until some time $t=t_{0}$ at which $x=\sigma$ where there is an impact

2010 Mathematics Subject Classification: Primary 34C23; Secondary 34C25, 37G15, $70 \mathrm{~K} 50$.

Keywords: periodic orbit, impact systems, persistence.

First author was partially supported by Grants VEGA-MS 1/0071/14 and VEGA-SAV $2 / 0029 / 13$.

Second author was supported by project No. CZ.1.07/2.3.00/30.0005 funded by European Regional Development Fund. 


\section{FEČKAN - M. POSPÍŠIL}

with a rigid obstacle. Then, at $t=t_{0}$, we suppose that $\left(x\left(t_{0}^{-}\right), \dot{x}\left(t_{0}^{-}\right)\right)$is mapped in zero time via an impact law to

$$
x\left(t_{0}^{+}\right)=x\left(t_{0}^{-}\right) \quad \text { and } \quad \dot{x}\left(t_{0}^{+}\right)=-(1+\varepsilon r) \dot{x}\left(t_{0}^{-}\right)
$$

where $x\left(t^{ \pm}\right)=\lim _{s \rightarrow t^{ \pm}} x(s), \dot{x}\left(t^{ \pm}\right)=\lim _{s \rightarrow t^{ \pm}} \dot{x}(s)$ and $0<1+\varepsilon r \leq 1$ is the Newton coefficient of restriction [4]. Another well-known piecewise linear impact system is a weakly damped and forced inverted pendulum given by equations [15]

$$
\begin{gathered}
\ddot{x}+\varepsilon \zeta \dot{x}-x=\varepsilon \mu \cos \omega t \quad \text { if } \quad|x(t)|<\sigma, \\
x\left(t^{+}\right)=x\left(t^{-}\right) \quad \text { and } \quad \dot{x}\left(t^{+}\right)=-(1+\varepsilon r) \dot{x}\left(t^{-}\right) \quad \text { if }\left|x\left(t^{-}\right)\right|=\sigma .
\end{gathered}
$$

We can study coupled (1.1) and (1.2) to get higher dimensional impact system. So a system for weakly forced impact oscillator consists of an ordinary differential equation

$$
\ddot{x}=f_{1}(x, \dot{x})+\varepsilon g_{1}(x, \dot{x}, t, \varepsilon, \mu)
$$

and an impact condition

$$
\dot{x}\left(t^{+}\right)=f_{2}\left(\dot{x}\left(t^{-}\right)\right)+\varepsilon g_{2}\left(x\left(t^{-}\right), \dot{x}\left(t^{-}\right), t, \varepsilon, \mu\right) \quad \text { if } \quad h\left(x\left(t^{-}\right)\right)=0 .
$$

The above equation rewritten as an evolution system has a form

$$
\begin{aligned}
\dot{x}_{1} & =x_{2} \\
\dot{x}_{2} & =f_{1}\left(x_{1}, x_{2}\right)+\varepsilon g_{1}\left(x_{1}, x_{2}, t, \varepsilon, \mu\right), \\
x_{2}\left(t^{+}\right)=f_{2}\left(x_{2}\left(t^{-}\right)\right) & +\varepsilon g_{2}\left(x_{1}\left(t^{-}\right), x_{2}\left(t^{-}\right), t, \varepsilon, \mu\right) \quad \text { if } \quad h\left(x_{1}\left(t^{-}\right)\right)=0 .
\end{aligned}
$$

In this paper, we shall investigate a more general case (see (2.1) and (2.2) ).

The simplest type of motion of impact oscillators is a periodic orbit. For $\varepsilon=0$, (1.1) and (1.2) possess impact periodic orbits. A natural question arises under which conditions on $\zeta$ and $\mu$ those periodic orbits persist for $\varepsilon \neq 0$ small. A mathematical theory of continuation/persistence of periodic orbits is well developed for smooth nonlinear dynamical systems (NDS). We refer the reader to books [6,8,11] for more references. On the other hand, recently many interesting results appeared on developing of an analogical theory for perturbed piecewisesmooth NDS [1,3, 4, 15, 18, and mechanical systems with impact conditions [4, 5 , 7, 10, 15, 17, 19 23. Again we refer the reader for more applications and details to these works. 
On the other hand, recently we have completed in series of papers 12 14 our study on persistence of periodic solutions for discontinuous NDS in any finite dimensional spaces for transverse cases when periodic orbits of unperturbed systems transversally crossed discontinuity levels. In addition, asymptotic properties as stability, instability and hyperbolicity of persisted periodic orbits have been also studied. In all these papers, we determined persistence conditions in terms of Poincaré-Andronov-Melnikov functions.

In this paper, we investigate the impact case by deriving impact PoincaréAndronov-Melnikov function for establishing persistence of impact periodic solutions. Motivated by examples (1.1) and (1.2), an illustrative planar discontinuous system is presented as well. We finish this paper by hinting further results on persistence of impact periodic solutions which could be done by combining this paper with our former ones [12 14. We emphasize that persistence of impact periodic solutions in such general settings like in this paper have been not yet done in previous works, since we have no restriction on dimensions of spatial variables and parameters.

\section{Preliminaries}

Let $\Omega \subset \mathbb{R}^{n}$ be an open set in $\mathbb{R}^{n}$ and $h(x)$ be a $C^{r}$-function on $\bar{\Omega}$, with $r \geq 3$. We set $\Omega_{0}:=\{x \in \Omega \mid h(x)=0\}, \Omega_{1}:=\Omega \backslash \Omega_{0}$. Let $f_{1} \in C_{b}^{r}(\bar{\Omega})$, $f_{2} \in C_{b}^{r}\left(\Omega_{0}, \Omega_{0}\right), g_{1} \in C_{b}^{r}\left(\bar{\Omega} \times \mathbb{R} \times \mathbb{R} \times \mathbb{R}^{p}\right), g_{2} \in C_{b}^{r}\left(\Omega_{0} \times \mathbb{R} \times \mathbb{R} \times \mathbb{R}^{p}\right)$ and $h \in C_{b}^{r}(\bar{\Omega}, \mathbb{R})$, i.e., the derivatives of $f_{1,2}, g_{1,2}$ and $h$ are uniformly continuous and bounded up to the $r$ th order, respectively. Furthermore, we suppose that $g_{1,2}$ are $T$-periodic in $t \in \mathbb{R}$ and 0 is a regular value of $h$. Let $\varepsilon, \alpha \in \mathbb{R}$ and $\mu \in \mathbb{R}^{p}, p \geq 1$ be parameters. We shall use the notation $\langle\cdot, \cdot\rangle$ and $\|\cdot\|$ for inner product in $\mathbb{R}^{n}$ and norm generated by it, respectively, and $\left[v_{1}, \ldots, v_{k}\right]$ for the linear span of vectors $v_{1}, \ldots, v_{k}$.

We say that a function $x(t)$ is a solution of an impact system

$$
\begin{aligned}
\dot{x}=f_{1}(x)+\varepsilon g_{1}(x, t, \varepsilon, \mu), & x \in \Omega_{1}, \\
x\left(t^{+}\right)=f_{2}\left(x\left(t^{-}\right)\right)+\varepsilon g_{2}\left(x\left(t^{-}\right), t, \varepsilon, \mu\right) & \text { if } \quad h\left(x\left(t^{-}\right)\right)=0,
\end{aligned}
$$

if it is piecewise $C^{1}$-smooth satisfying equation (2.1) on $\Omega_{1}$, equation (2.2) on $\Omega_{0}$ and, moreover, the following holds: if for some $t_{0}$ we have $x\left(t_{0}\right) \in \Omega_{0}$, then there exists $\rho>0$ such that for any $t \in\left(t_{0}-\rho, t_{0}\right), s \in\left(t_{0}, t_{0}+\rho\right)$ we have $h(x(t)) h(x(s))>0$. 


\section{Poincaré-Andronov-Melnikov function}

In this section we investigate the persistence of a single $T$-periodic orbit of autonomous system with impact under nonautonomous perturbation and derive a sufficient condition. A modelling problem is given by (2.1)-(2.2) for which we assume

H1) The unperturbed equation

$$
\dot{x}=f_{1}(x)
$$

has a $T$-periodic orbit $\gamma(t)$ which is discontinuous at $t=t_{1} \in(0, T)$ where it satisfies impact condition

$$
x\left(t^{+}\right)=f_{2}\left(x\left(t^{-}\right)\right) \quad \text { if } \quad h\left(x\left(t^{-}\right)\right)=0 .
$$

The orbit is given by its initial point $x_{0} \in \Omega_{1}$ and consists of two branches

$$
\gamma(t)= \begin{cases}\gamma_{1}(t) & \text { if } t \in\left[0, t_{1}\right), \\ \left\{x_{1}, x_{2}\right\} & \text { if } t=t_{1}, \\ \gamma_{2}(t) & \text { if } t \in\left(t_{1}, T\right]\end{cases}
$$

where $0<t_{1}<T, \gamma(t) \in \Omega_{1}$ for $t \in\left[0, t_{1}\right) \cup\left(t_{1}, T\right], \gamma\left(t_{1}\right) \subset \Omega_{0}$ and

$$
\begin{aligned}
x_{1} & :=\gamma_{1}\left(t_{1}^{-}\right) \in \Omega_{0}, \\
x_{2} & :=\gamma_{2}\left(t_{1}^{+}\right) \in \Omega_{0}, \\
x_{0} & :=\gamma_{2}(T)=\gamma_{1}(0) \in \Omega_{1} .
\end{aligned}
$$

H2) Moreover, we also assume that

$$
\operatorname{Dh}\left(x_{1}\right) f_{1}\left(x_{1}\right) \operatorname{Dh}\left(x_{2}\right) f_{1}\left(x_{2}\right)<0 .
$$

The geometric meaning of assumption $\mathrm{H} 2$ ) is that the impact periodic solution $\gamma(t)$ from H1) transversally hits and leaves the impact surface $\Omega_{0}$ at $t_{1}^{-}$and $t_{1}^{+}$, respectively.

Next, since impact system (3.1) -3.2) is autonomous, $\gamma(t-\alpha)$ is also its solution for any $\alpha \in \mathbb{R}$. So we are looking for a forced $T$-periodic solution $x(t)$ of the perturbed impact system (2.1) (2.2) which is orbitally close to $\gamma$, i.e., $x(t) \sim \gamma(t-\alpha)$ for some $\alpha$ depending on $\varepsilon \neq 0$ small. For this reason, by shifting the time, we study a shifted (2.1) $-(2.2)$ of the form

$$
\begin{array}{cl}
\dot{x}=f_{1}(x)+\varepsilon g_{1}(x, t+\alpha, \varepsilon, \mu), & x \in \Omega_{1}, \\
x\left(t^{+}\right)=f_{2}\left(x\left(t^{-}\right)\right)+\varepsilon g_{2}\left(x\left(t^{-}\right), t+\alpha, \varepsilon, \mu\right) & \text { if } h\left(x\left(t^{-}\right)\right)=0
\end{array}
$$

with additional parameter $\alpha \in \mathbb{R}$. 
Let $x(\tau, \xi)(t, \varepsilon, \mu, \alpha)$ denote the solution of initial value problem

$$
\begin{aligned}
\dot{x} & =f_{1}(x)+\varepsilon g_{1}(x, t+\alpha, \varepsilon, \mu) \\
x(\tau) & =\xi .
\end{aligned}
$$

First, we modify our result from [12] for impact system (3.5), (3.6).

Lemma 3.1. Assume $\mathrm{H} 1$ ) and $\mathrm{H} 2)$. Then there exist $\varepsilon_{0}, r_{0}>0$ and a Poincaré mapping (cf. Fig. 1)

$$
P(\cdot, \varepsilon, \mu, \alpha): B\left(x_{0}, r_{0}\right) \rightarrow \Sigma
$$

for all fixed $\varepsilon \in\left(-\varepsilon_{0}, \varepsilon_{0}\right), \mu \in \mathbb{R}^{p}, \alpha \in \mathbb{R}$, where $B(x, r)$ is a ball in $\mathbb{R}^{n}$ with center at $x$ and radius $r$, and

$$
\Sigma=\left\{y \in \mathbb{R}^{n} \mid\left\langle y-x_{0}, f_{1}\left(x_{0}\right)\right\rangle=0\right\} .
$$

Moreover, $P: B\left(x_{0}, r_{0}\right) \times\left(-\varepsilon_{0}, \varepsilon_{0}\right) \times \mathbb{R}^{p} \times \mathbb{R} \rightarrow \mathbb{R}^{n}$ is $C^{r}$-smooth in all arguments and $B\left(x_{0}, r_{0}\right) \subset \Omega_{1}$.

P r o of. The lemma follows from Implicit Function Theorem [9]. We obtain the existence of positive constants $\tau_{1}, r_{1}, \delta_{1}, \varepsilon_{1}$ and $C^{r}$-function

$$
t_{1}(\cdot, \cdot, \cdot, \cdot, \cdot):\left(-\tau_{1}, \tau_{1}\right) \times B\left(x_{0}, r_{1}\right) \times\left(-\varepsilon_{1}, \varepsilon_{1}\right) \times \mathbb{R}^{p} \times \mathbb{R} \rightarrow\left(t_{1}-\delta_{1}, t_{1}+\delta_{1}\right)
$$

such that $h(x(\tau, \xi)(t, \varepsilon, \mu, \alpha))=0$ for $\tau \in\left(-\tau_{1}, \tau_{1}\right), \xi \in B\left(x_{0}, r_{1}\right) \subset \Omega_{1}, \varepsilon \in$ $\left(-\varepsilon_{1}, \varepsilon_{1}\right), \mu \in \mathbb{R}^{p}, \alpha \in \mathbb{R}$ and $t \in\left(t_{1}-\delta_{1}, t_{1}+\delta_{1}\right)$ if and only if $t=t_{1}(\tau, \xi, \varepsilon, \mu, \alpha)$. Moreover, $t_{1}\left(0, x_{0}, 0, \mu, \alpha\right)=t_{1}$.

Analogically, we derive function $t_{2}$ satisfying

$$
\begin{aligned}
& \left\langlex \left( t_{1}(\tau, \xi, \varepsilon, \mu, \alpha), f_{2}\left(x(\tau, \xi)\left(t_{1}(\tau, \xi, \varepsilon, \mu, \alpha), \varepsilon, \mu, \alpha\right)\right)\right.\right. \\
& \left.+\varepsilon g_{2}\left(x(\tau, \xi)\left(t_{1}(\tau, \xi, \varepsilon, \mu, \alpha), \varepsilon, \mu, \alpha\right), t_{1}(\tau, \xi, \varepsilon, \mu, \alpha)+\alpha, \varepsilon, \mu\right)\right) \\
& \left.\left(t_{2}(\tau, \xi, \varepsilon, \mu, \alpha), \varepsilon, \mu, \alpha\right)-x_{0}, f_{1}\left(x_{0}\right)\right\rangle=0 .
\end{aligned}
$$

Moreover, we have $t_{2}\left(0, x_{0}, 0, \mu, \alpha\right)=T$. Poincaré mapping is then defined as

$$
\begin{aligned}
P(\xi, \varepsilon, \mu, \alpha)=x & \left(t_{1}(0, \xi, \varepsilon, \mu, \alpha), f_{2}\left(x(0, \xi)\left(t_{1}(0, \xi, \varepsilon, \mu, \alpha), \varepsilon, \mu, \alpha\right)\right)\right. \\
& \left.+\varepsilon g_{2}\left(x(0, \xi)\left(t_{1}(0, \xi, \varepsilon, \mu, \alpha), \varepsilon, \mu, \alpha\right), t_{1}(0, \xi, \varepsilon, \mu, \alpha)+\alpha, \varepsilon, \mu\right)\right) \\
& \left(t_{2}(0, \xi, \varepsilon, \mu, \alpha), \varepsilon, \mu, \alpha\right) .
\end{aligned}
$$

The proof is finished.

Since we are looking for persisting periodic orbit with fixed period $T$, we have to solve a couple of equations

$$
\begin{aligned}
P(\xi, \varepsilon, \mu, \alpha) & =\xi, \\
t_{2}(0, \xi, \varepsilon, \mu, \alpha) & =T .
\end{aligned}
$$




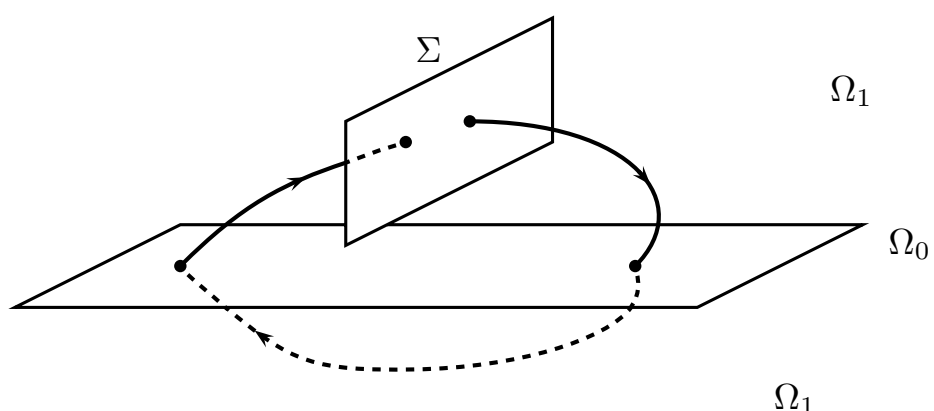

Figure 1. Impact Poincaré mapping

Therefore, we define so-called stroboscopic Poincaré mapping (cf. [4,12])

$$
\begin{aligned}
& \widetilde{P}(\xi, \varepsilon, \mu, \alpha) \\
= & x\left(t_{1}(0, \xi, \varepsilon, \mu, \alpha), f_{2}\left(x(0, \xi)\left(t_{1}(0, \xi, \varepsilon, \mu, \alpha), \varepsilon, \mu, \alpha\right)\right)\right. \\
& \left.+\varepsilon g_{2}\left(x(0, \xi)\left(t_{1}(0, \xi, \varepsilon, \mu, \alpha), \varepsilon, \mu, \alpha\right), t_{1}(0, \xi, \varepsilon, \mu, \alpha)+\alpha, \varepsilon, \mu\right)\right)(T, \varepsilon, \mu, \alpha)
\end{aligned}
$$

and solve a single equation

$$
F(\xi, \varepsilon, \mu, \alpha):=\xi-\widetilde{P}(\xi, \varepsilon, \mu, \alpha)=0 \quad \text { for } \quad \xi \in \Sigma .
$$

Now, we calculate the linearization of $\widetilde{P}$ at $(\xi, \varepsilon)=\left(x_{0}, 0\right)$ analogically to [12]. We obtain the next result.

Lemma 3.2. Let $\widetilde{P}(\xi, \varepsilon, \mu, \alpha)$ be defined by (3.9). Then for all $\mu \in \mathbb{R}^{p}, \alpha \in \mathbb{R}$

$$
\begin{aligned}
& \widetilde{P}_{\xi}\left(x_{0}, 0, \mu, \alpha\right)=A(0) \\
& \widetilde{P}_{\varepsilon}\left(x_{0}, 0, \mu, \alpha\right)=\int_{0}^{T} A(s) g_{1}(\gamma(s), s+\alpha, 0, \mu) \mathrm{d} s+X_{2}(T) g_{2}\left(x_{1}, t_{1}+\alpha, 0, \mu\right),
\end{aligned}
$$

where $\widetilde{P}_{\xi}, \widetilde{P}_{\varepsilon}$ are partial derivatives of $\widetilde{P}$ with respect to $\xi$, $\varepsilon$, respectively. Here $A(t)$ is given by

$$
A(t)= \begin{cases}X_{2}(T) S X_{1}\left(t_{1}\right) X_{1}^{-1}(t) & \text { if } t \in\left[0, t_{1}\right) \\ X_{2}(T) X_{2}^{-1}(t) & \text { if } t \in\left[t_{1}, T\right]\end{cases}
$$

with impact saltation matrix

$$
S=\mathrm{D} f_{2}\left(x_{1}\right)+\frac{\left(f_{1}\left(x_{2}\right)-\mathrm{D} f_{2}\left(x_{1}\right) f_{1}\left(x_{1}\right)\right) \operatorname{Dh}\left(x_{1}\right)}{\operatorname{Dh}\left(x_{1}\right) f_{1}\left(x_{1}\right)}
$$


and fundamental matrix solutions $X_{1}(t), X_{2}(t)$ satisfying, respectively,

$$
\begin{aligned}
\dot{X}_{1}(t) & =\mathrm{D} f_{1}(\gamma(t)) X_{1}(t) & \dot{X}_{2}(t) & =\mathrm{D} f_{1}(\gamma(t)) X_{2}(t) \\
X_{1}(0) & =\mathbb{I}, & X_{2}\left(t_{1}\right) & =\mathbb{I} .
\end{aligned}
$$

In addition, $\widetilde{P}_{\xi}\left(x_{0}, 0, \mu, \alpha\right)$ has an eigenvalue 1 with corresponding eigenvector $f_{1}\left(x_{0}\right)$, i.e.,

$$
\widetilde{P}_{\xi}\left(x_{0}, 0, \mu, \alpha\right) f_{1}\left(x_{0}\right)=f_{1}\left(x_{0}\right) .
$$

P r o of. The statement on the derivatives of $\widetilde{P}$ follows from its definition with the aid of the following identities

for $t \in\left[0, t_{1}\right]$,

$$
\begin{aligned}
& \mathrm{D}_{\xi} x\left(0, x_{0}\right)(t, 0, \mu, \alpha)=X_{1}(t) \\
& \mathrm{D}_{\varepsilon} x\left(0, x_{0}\right)(t, 0, \mu, \alpha)=\int_{0}^{t} X_{1}(t) X_{1}^{-1}(s) g_{1}(\gamma(s), s+\alpha, 0, \mu) \mathrm{d} s \\
& \left.0, t_{1}\right]
\end{aligned}
$$

$$
\begin{aligned}
& \mathrm{D}_{\xi} x\left(t_{1}, x_{2}\right)(t, 0, \mu, \alpha)=X_{2}(t) \\
& \mathrm{D}_{\tau} x\left(t_{1}, x_{2}\right)(t, 0, \mu, \alpha)=-X_{2}(t) f_{1}\left(x_{2}\right) \\
& \mathrm{D}_{\varepsilon} x\left(t_{1}, x_{2}\right)(t, 0, \mu, \alpha)=\int_{t_{1}}^{t} X_{2}(t) X_{2}^{-1}(s) g_{1}(\gamma(s), s+\alpha, 0, \mu) \mathrm{d} s
\end{aligned}
$$

\[ \mathrm{D}_{\varepsilon} x\left(t_{1}, x_{2}\right)(t, 0, \mu, \alpha)=\int_{t_{1}}^{t} X_{2}(t) X_{2}^{-1}(s) g_{1}(\gamma(s), s+\alpha, 0, \mu) \mathrm{d} s \]
for $t \in\left[t_{1}, T\right]$ and

$$
\begin{aligned}
\mathrm{D}_{\xi} t_{1}\left(0, x_{0}, 0, \mu, \alpha\right) & =-\frac{\mathrm{D} h\left(x_{1}\right) X_{1}\left(t_{1}\right)}{\operatorname{Dh}\left(x_{1}\right) f_{1}\left(x_{1}\right)}, \\
\mathrm{D}_{\varepsilon} t_{1}\left(0, x_{0}, 0, \mu, \alpha\right) & =-\frac{\operatorname{Dh}\left(x_{1}\right) \int_{0}^{t_{1}} X_{1}\left(t_{1}\right) X_{1}^{-1}(s) g_{1}(\gamma(s), s+\alpha, 0, \mu) \mathrm{d} s}{\operatorname{Dh}\left(x_{1}\right) f_{1}\left(x_{1}\right)} .
\end{aligned}
$$

To proceed with the proof, we note $\varepsilon=0$ results $x(\tau, \xi)(t, 0, \mu, \alpha)=x(\tau, \xi)(t)$, the solution of $\dot{x}=f_{1}(x), x(\tau)=\xi$, and it is independent of $(\mu, \alpha)$. Analogically $t_{1}(\tau, \xi, 0, \mu, \alpha)=t_{1}(\tau, \xi), t_{2}(\tau, \xi, 0, \mu, \alpha)=t_{2}(\tau, \xi), P(\xi, 0, \mu, \alpha)=P(\xi)$ and $\widetilde{P}(\xi, 0, \mu, \alpha)=\widetilde{P}(\xi)$.

Now, since for all $t>0$ small, it holds that

$$
x\left(0, \gamma_{1}(t)\right)\left(t_{1}\left(0, \gamma_{1}(t)\right)\right)=x\left(0, x_{0}\right)\left(t+t_{1}\left(0, \gamma_{1}(t)\right)\right)=\gamma_{1}\left(t+t_{1}\left(0, \gamma_{1}(t)\right)\right)
$$

is an element of $\Omega_{0}$ and as well of $\{\gamma(t) \mid t \in \mathbb{R}\}$ we have

$$
t+t_{1}\left(0, \gamma_{1}(t)\right)=t_{1}
$$

Consequently

$$
x\left(0, \gamma_{1}(t)\right)\left(t_{1}\left(0, \gamma_{1}(t)\right)\right)=x_{1}
$$


Then we obtain

$$
\begin{aligned}
\widetilde{P}\left(\gamma_{1}(t)\right) & =x\left(t_{1}\left(0, \gamma_{1}(t)\right), f_{2}\left(x\left(0, \gamma_{1}(t)\right)\left(t_{1}\left(0, \gamma_{1}(t)\right)\right)\right)\right)(T) \\
& =x\left(t_{1}\left(0, \gamma_{1}(t)\right), f_{2}\left(x_{1}\right)\right)(T) \\
& =x\left(t_{1}-t, x_{2}\right)(T)=x\left(t_{1}, x_{2}\right)(T+t)=\gamma_{1}(t)
\end{aligned}
$$

Hence

$$
\widetilde{P}_{\xi}\left(x_{0}, 0, \mu, \alpha\right) f_{1}\left(x_{0}\right)=\mathrm{D}_{t}\left[\widetilde{P}\left(\gamma_{1}(t)\right)\right]_{t=0^{+}}=\mathrm{D}_{t}\left[\gamma_{1}(t)\right]_{t=0^{+}}=f_{1}\left(x_{0}\right) .
$$

The proof is finished.

We solve equation (3.10) for $(\xi, \alpha) \in \Sigma \times \mathbb{R}$ with parameters $\varepsilon$, $\mu$ using Lyapunov-Schmidt reduction method [6]. Obviously $F\left(x_{0}, 0, \mu, \alpha\right)=0$ for all $(\mu, \alpha) \in \mathbb{R}^{p} \times \mathbb{R}$. Let us denote

$$
Z=\mathcal{N} \mathrm{D}_{\xi} F\left(x_{0}, 0, \mu, \alpha\right), \quad Y=\mathcal{R} \mathrm{D}_{\xi} F\left(x_{0}, 0, \mu, \alpha\right)
$$

the null space and the range of the corresponding operator and

$$
\mathcal{Q}: \mathbb{R}^{n} \rightarrow Y, \quad \mathcal{P}: \mathbb{R}^{n} \rightarrow Y^{\perp}
$$

orthogonal projections onto $Y$ and $Y^{\perp}$, respectively, where $Y^{\perp}$ is the orthogonal complement to $Y$ in $\mathbb{R}^{n}$. Here we take the third assumption

H3) $\mathcal{N} D_{\xi} F\left(x_{0}, 0, \mu, \alpha\right)=\left[f_{1}\left(x_{0}\right)\right]$.

Equation (3.10) is split into couple of equations

$$
\begin{aligned}
& \mathcal{Q} F(\xi, \varepsilon, \mu, \alpha)=0, \\
& \mathcal{P} F(\xi, \varepsilon, \mu, \alpha)=0
\end{aligned}
$$

where the first one can be solved using Implicit Function Theorem, since

$$
\mathcal{Q} F\left(x_{0}, 0, \mu, \alpha\right)=0
$$

and $\mathcal{Q} \mathrm{D}_{\xi} F\left(x_{0}, 0, \mu, \alpha\right)$ is an isomorphism from $\left[f_{1}\left(x_{0}\right)\right]^{\perp}$ onto $Y$ for all $(\mu, \alpha) \in$ $\mathbb{R}^{p} \times \mathbb{R}$. Thus we get the existence of a $C^{r}$-function $\xi=\xi(\varepsilon, \mu, \alpha)$ for $\varepsilon$ close to 0 and $(\mu, \alpha) \in \mathbb{R}^{p} \times \mathbb{R}$ such that $\mathcal{Q} F(\xi(\varepsilon, \mu, \alpha), \varepsilon, \mu, \alpha)=0$ for all such $(\varepsilon, \mu, \alpha)$ and $\xi(0, \mu, \alpha)=x_{0}$. The second equation is so-called persistence equation for $\alpha \in \mathbb{R}$

$$
\mathcal{P} F(\xi(\varepsilon, \mu, \alpha), \varepsilon, \mu, \alpha)=0 .
$$

Let $\psi \in Y^{\perp}$ be arbitrary and fixed. Then we can write

$$
\mathcal{P} u=\frac{\langle u, \psi\rangle \psi}{\|\psi\|^{2}}
$$


and persistence equation (3.19) has the form

$$
G(\varepsilon, \mu, \alpha):=\frac{\langle F(\xi(\varepsilon, \mu, \alpha), \varepsilon, \mu, \alpha), \psi\rangle \psi}{\|\psi\|^{2}}=0 .
$$

Clearly $G(0, \mu, \alpha)=0$ for all $(\mu, \alpha) \in \mathbb{R}^{p} \times \mathbb{R}$. Moreover, we want the periodic orbit to persist, so we need to solve $G(\varepsilon, \mu, \alpha)=0$ for $\varepsilon \neq 0$ small. But since $G(\varepsilon, \mu, \alpha)=\mathrm{D}_{\varepsilon} G(0, \mu, \alpha) \varepsilon+o(\varepsilon)$, the equality $\mathrm{D}_{\varepsilon} G\left(0, \mu_{0}, \alpha_{0}\right)=0$ is a necessary condition for a point $\left(0, \mu_{0}, \alpha_{0}\right)$ to be a starting persistence value, this means if there is a sequence $\left\{\left(\varepsilon_{n}, \mu_{n}, \alpha_{n}\right)\right\}_{n \in \mathbb{N}}$ such that $\varepsilon_{n} \neq 0,\left(\varepsilon_{n}, \mu_{n}, \alpha_{n}\right) \rightarrow\left(0, \mu_{0}, \alpha_{0}\right)$ and $G\left(\varepsilon_{n}, \mu_{n}, \alpha_{n}\right)=0$, then $\mathrm{D}_{\varepsilon} G\left(0, \mu_{0}, \alpha_{0}\right)=0$. So we derive

$$
\begin{aligned}
\mathrm{D}_{\varepsilon} G(0, \mu, \alpha) & =\frac{\left\langle\left(\mathrm{D}_{\xi} F\left(x_{0}, 0, \mu, \alpha\right) \mathrm{D}_{\varepsilon} \xi(0, \mu, \alpha)+\mathrm{D}_{\varepsilon} F\left(x_{0}, 0, \mu, \alpha\right)\right), \psi\right\rangle \psi}{\|\psi\|^{2}} \\
& =\frac{\left\langle\mathrm{D}_{\varepsilon} F\left(x_{0}, 0, \mu, \alpha\right), \psi\right\rangle \psi}{\|\psi\|^{2}}=-\frac{\left\langle\mathrm{D}_{\varepsilon} \widetilde{P}\left(x_{0}, 0, \mu, \alpha\right), \psi\right\rangle \psi}{\|\psi\|^{2}} .
\end{aligned}
$$

We denote

$$
M^{\mu}(\alpha)=\int_{0}^{T}\left\langle g_{1}(\gamma(s), s+\alpha, 0, \mu), A^{*}(s) \psi\right\rangle \mathrm{d} s+\left\langle X_{2}(T) g_{2}\left(x_{1}, t_{1}+\alpha, 0, \mu\right), \psi\right\rangle
$$

the impact Poincaré-Andronov-Melnikov function, where

$$
A^{*}(t)= \begin{cases}X_{1}^{-1 *}(t) X_{1}^{*}\left(t_{1}\right) S^{*} X_{2}^{*}(T) & \text { if } t \in\left[0, t_{1}\right) \\ X_{2}^{-1 *}(t) X_{2}^{*}(T) & \text { if } t \in\left[t_{1}, T\right] .\end{cases}
$$

Note that $\mathrm{D}_{\varepsilon} G(0, \mu, \alpha)=-\frac{M^{\mu}(\alpha) \psi}{\|\psi\|^{2}}$.

Linearization of unperturbed impact system (3.1)-(3.2) along $T$-periodic solution $\gamma(t)$ gives the variational equation

$$
\dot{x}(t)=\mathrm{D} f_{1}(\gamma(t)) x(t)
$$

with impulsive condition

$$
x\left(t_{1}^{+}\right)=S x\left(t_{1}^{-}\right)
$$

and periodic condition

$$
B(x(0)-x(T))=0
$$

where $B=\frac{\psi \psi^{*}}{\|\psi\|^{2}}$ is the orthogonal projection onto $Y^{\perp}$. From definition of $X_{1}(t), X_{2}(t)$

$$
X(t)= \begin{cases}X_{1}(t) & \text { if } t \in\left[0, t_{1}\right) \\ X_{2}(t) S X_{1}\left(t_{1}\right) & \text { if } t \in\left[t_{1}, T\right]\end{cases}
$$




\section{FEČKAN - M. POSPÍŠIL}

solves variational equation (3.23) and conditions (3.24), 3.25). Now, we recall a classical result (cf. [16]) and our result (see [13]).

Lemma 3.3. Let $X(t)$ be a fundamental matrix solution of equation $\dot{X}=U X$. Then $X^{-1 *}(t)$ is a fundamental matrix solution of adjoint equation $\dot{Y}=-U^{*} Y$.

LemMA 3.4. Let $A(t) \in C\left([0, T], L\left(\mathbb{R}^{n}\right)\right), B_{1}, B_{2}, B_{3} \in L\left(\mathbb{R}^{n}\right), 0<t_{1}<t_{2}<T$ and $h \in \mathcal{C}:=C\left(\left[0, t_{1}\right], \mathbb{R}^{n}\right) \cap C\left(\left[t_{1}, t_{2}\right], \mathbb{R}^{n}\right) \cap C\left(\left[t_{2}, T\right], \mathbb{R}^{n}\right)$. Then the nonhomogeneous problem

$$
\begin{aligned}
\dot{x} & =A(t) x+h(t), \\
x\left(t_{i}+\right) & =B_{i} x\left(t_{i}-\right), \quad i=1,2, \\
B_{3}(x(T)-x(0)) & =0
\end{aligned}
$$

has a solution $x \in \mathcal{C}^{1}:=C^{1}\left(\left[0, t_{1}\right], \mathbb{R}^{n}\right) \cap C^{1}\left(\left[t_{1}, t_{2}\right], \mathbb{R}^{n}\right) \cap C^{1}\left(\left[t_{2}, T\right], \mathbb{R}^{n}\right)$ if and only if $\int_{0}^{T}\langle h(t), v(t)\rangle \mathrm{d} t=0$ for any solution $v \in \mathcal{C}^{1}$ of the adjoint system given by

$$
\begin{aligned}
\dot{v} & =-A^{*}(t) v, \\
v\left(t_{i}-\right) & =B_{i}^{*} v\left(t_{i}+\right), \quad i=1,2, \\
v(T) & =v(0) \in \mathcal{N} B_{3}^{\perp} .
\end{aligned}
$$

On letting $B_{1}=S, B_{2}=\mathbb{I}, B_{3}=B$ in the last lemma one can see that the adjoint variational system of (3.1) and impact condition (3.2) (i.e., adjoint system of (3.23)-(3.25) ) is given by the following linear impulsive boundary value problem

$$
\begin{aligned}
\dot{x}(t) & =-\mathrm{D} f_{1}^{*}(\gamma(t)) x(t), \quad t \in[0, T], \\
x\left(t_{1}^{-}\right) & =S^{*} x\left(t_{1}^{+}\right), \\
x(T) & =x(0) \in Y^{\perp} .
\end{aligned}
$$

From (3.22) we know that $A^{*}(t) \psi$ solves adjoint variational equation with impulsive condition. To see that it satisfies the boundary condition as well, we consider

$$
0=\left\langle\mathrm{D}_{\xi} F\left(x_{0}, 0, \mu, \alpha\right) \xi, \psi\right\rangle=\langle(\mathbb{I}-A(0)) \xi, \psi\rangle=\left\langle\xi,\left(\mathbb{I}-A^{*}(0)\right) \psi\right\rangle
$$

for all $\xi \in\left[f_{1}\left(x_{0}\right)\right]^{\perp}$ and if $\xi \in\left[f_{1}\left(x_{0}\right)\right]$, from Lemma 3.2 follows

$$
0=\langle\xi-\xi, \psi\rangle=\langle(\mathbb{I}-A(0)) \xi, \psi\rangle=\left\langle\xi,\left(\mathbb{I}-A^{*}(0)\right) \psi\right\rangle .
$$

As a consequence, we can take in (3.21) any solution of the adjoint variational system (3.26). Summarizing, we get the main result. 
TheOREm 3.5. Let $\psi \in Y^{\perp}$ be arbitrary and fixed, $Y$ be given by (3.16) and $A^{*}(t), M^{\mu}(\alpha)$ be defined by (3.22), (3.21), respectively. If $\alpha_{0}$ is a simple root of function $M^{\mu_{0}}(\alpha)$, i.e.,

$$
\begin{aligned}
& \int_{0}^{T}\left\langle g_{1}\left(\gamma(s), s+\alpha_{0}, 0, \mu_{0}\right), A^{*}(s) \psi\right\rangle \mathrm{d} s+\left\langle X_{2}(T) g_{2}\left(x_{1}, t_{1}+\alpha_{0}, 0, \mu_{0}\right), \psi\right\rangle=0 \\
& \int_{0}^{T}\left\langle\mathrm{D}_{t} g_{1}\left(\gamma(s), s+\alpha_{0}, 0, \mu_{0}\right), A^{*}(s) \psi\right\rangle \mathrm{d} s+\left\langle X_{2}(T) \mathrm{D}_{t} g_{2}\left(x_{1}, t_{1}+\alpha_{0}, 0, \mu_{0}\right), \psi\right\rangle \neq 0
\end{aligned}
$$

then there exists a unique $C^{r-1}$-function $\alpha(\varepsilon, \mu)$ for $\varepsilon \sim 0$ small and $\mu \sim \mu_{0}$ such that $\alpha\left(0, \mu_{0}\right)=\alpha_{0}$ and there is a unique T-periodic solution $x_{\varepsilon, \mu}(t)$ of equation (2.1) with parameters $\varepsilon \neq 0$ sufficiently small, $\mu$ close to $\mu_{0}$ and $\alpha=\alpha(\varepsilon, \mu)$, which satisfies condition (2.2) and $\left|x_{\varepsilon, \mu}(t)-\gamma(t-\alpha(\varepsilon, \mu))\right|=O(\varepsilon)$.

Pr o of. We set

$$
H(\varepsilon, \mu, \alpha)= \begin{cases}\mathrm{D}_{\varepsilon} G(0, \mu, \alpha) & \text { for } \varepsilon=0, \\ G(\varepsilon, \mu, \alpha) / \varepsilon & \text { for } \varepsilon \neq 0 .\end{cases}
$$

Then $H$ is $C^{r-1}$-smooth. Assumptions of our theorem imply $H\left(0, \mu_{0}, \alpha_{0}\right)=0$ and $\mathrm{D}_{\alpha} H\left(0, \mu_{0}, \alpha_{0}\right) \neq 0$. By Implicit Function Theorem there exists a unique $C^{r-1}$-function $\alpha(\varepsilon, \mu)$ for $\varepsilon \sim 0$ small and $\mu \sim \mu_{0}$ such that $\alpha\left(0, \mu_{0}\right)=\alpha_{0}$, and $H(\varepsilon, \mu, \alpha(\varepsilon, \mu))=0$. But this means that $x(0, \xi(\varepsilon, \mu, \alpha(\varepsilon, \mu)))(t, \varepsilon, \mu, \alpha(\varepsilon, \mu))$ is a solution of (3.5) $-(3.6)$ with $\alpha=\alpha(\varepsilon, \mu)$ and it satisfies

$$
|x(0, \xi(\varepsilon, \mu, \alpha(\varepsilon, \mu)))(t, \varepsilon, \mu, \alpha(\varepsilon, \mu))-\gamma(t)|=O(\varepsilon) .
$$

Then

$$
x_{\varepsilon, \mu}(t)=x(0, \xi(\varepsilon, \mu, \alpha(\varepsilon, \mu)))(t-\alpha(\varepsilon, \mu), \varepsilon, \mu, \alpha(\varepsilon, \mu))
$$

is the desired solution of (2.1) $-(2.2)$. The proof is completed.

\section{Pendulum hitting moving obstacle}

Here we provide an application of derived theory to problem of mathematical pendulum which impacts an oscillating wall (see Fig. 2). The horizontal distance between the wall and the center of the pendulum is $\delta+\varepsilon z(t, \varepsilon, \mu)$ where $z$ is periodic in $t$ and $\delta$ is a positive constant. We denote $x$ the angle and $l$ the length of the massless cord. Then $x$ satisfies the dimensionless equation

$$
\ddot{x}=-\omega^{2} x
$$


M. FEČKAN - M. POSPÍŠIL

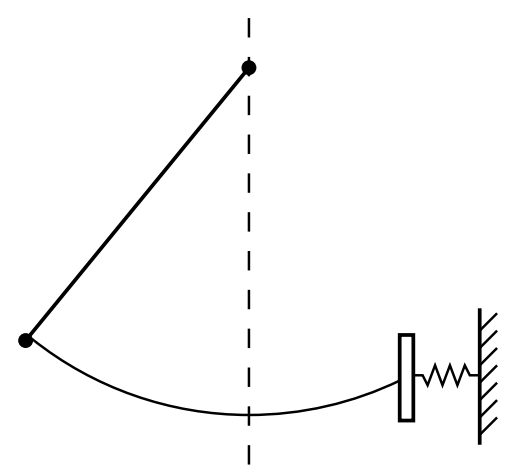

FiguRE 2. Impacting pendulum

with a given frequency $\omega>0$ and impact condition

$$
\dot{x}\left(t^{+}\right)=-\dot{x}\left(t^{-}\right)+\varepsilon \dot{z}\left(t^{-}, \varepsilon, \mu\right) \frac{\sqrt{l^{2}-\left(\delta+\varepsilon z\left(t^{-}, \varepsilon, \mu\right)\right)^{2}}}{l}
$$

whenever

$$
x\left(t^{-}\right)-\arcsin \frac{\delta+\varepsilon z\left(t^{-}, \varepsilon, \mu\right)}{l}=0,
$$

which follows from actual position of the wall and its speed projected onto tangent line to the trajectory of bob. Writing as a system we get

$$
\begin{aligned}
& \dot{x}=\omega y \\
& \dot{y}=-\omega x
\end{aligned}
$$

and

$$
\begin{aligned}
& x\left(t^{+}\right)=x\left(t^{-}\right) \\
& y\left(t^{+}\right)=-y\left(t^{-}\right)+\varepsilon \dot{z}\left(t^{-}, \varepsilon, \mu\right) \frac{\sqrt{l^{2}-\left(\delta+\varepsilon z\left(t^{-}, \varepsilon, \mu\right)\right)^{2}}}{\omega l}
\end{aligned}
$$

if

$$
x\left(t^{-}\right)-\arcsin \frac{\delta+\varepsilon z\left(t^{-}, \varepsilon, \mu\right)}{l}=0 .
$$

To obtain a problem in form of (3.5), (3.6) we introduce parameter $\alpha$ and transform the variables

$$
u=x-\arcsin \frac{\delta+\varepsilon z(t+\alpha, \varepsilon, \mu)}{l}+\arcsin \frac{\delta}{l}, \quad v=y .
$$


So we get

$$
\begin{aligned}
& \dot{u}(t)=\omega v(t)-\varepsilon \frac{\dot{z}(t+\alpha, \varepsilon, \mu)}{\sqrt{l^{2}-(\delta+\varepsilon z(t+\alpha, \varepsilon, \mu))^{2}}} \\
& \dot{v}(t)=-\omega u(t)-\varepsilon \frac{\omega z(t+\alpha, 0, \mu)}{\sqrt{l^{2}-\delta^{2}}}+O\left(\varepsilon^{2}\right)
\end{aligned}
$$

with impact condition

$$
\begin{aligned}
& u\left(t^{+}\right)=u\left(t^{-}\right), \\
& v\left(t^{+}\right)=-v\left(t^{-}\right)+\varepsilon \dot{z}\left(t^{-}+\alpha, \varepsilon, \mu\right) \frac{\sqrt{l^{2}-\left(\delta+\varepsilon z\left(t^{-}+\alpha, \varepsilon, \mu\right)\right)^{2}}}{\omega l} \\
& \text { if } h\left(u\left(t^{-}\right), v\left(t^{-}\right)\right)=0
\end{aligned}
$$

where

$$
h(u, v)=u-\arcsin \frac{\delta}{l} .
$$

We add the lower index $\varepsilon$ to denote the dependence on $\varepsilon$ in (4.1), (4.2), i.e., (4.1) 0 , (4.2) 0 shall denote the unperturbed system and the unperturbed impact condition, respectively. Moreover, we denote $\hat{u}=\arcsin \frac{\delta}{l}$.

In this case we have $\Omega_{0}=\left\{(u, v) \in \mathbb{R}^{2} \mid u=\hat{u}\right\}, \Sigma=\left\{(u, 0) \in \mathbb{R}^{2} \mid u<-\hat{u}\right\}$ and the following lemma describing the unperturbed problem.

Lemma 4.1. System (4.1) 0 , (4.2) 0 possesses a family of periodic orbits $\gamma^{u}(t)$ parametrized by $u<-\hat{u}$ such that

$$
\gamma^{u}(t)= \begin{cases}(u \cos \omega t,-u \sin \omega t) & \text { if } t \in\left[0, t_{1}\right), \\ \left\{\left(u_{1}, v_{1}\right),\left(u_{2}, v_{2}\right)\right\} & \text { if } t=t_{1}, \\ (u \cos \omega(T-t), u \sin \omega(T-t)) & \text { if } t \in\left(t_{1}, T\right]\end{cases}
$$

where

$$
\begin{aligned}
t_{1} & =\frac{1}{\omega} \arccos \frac{\hat{u}}{u}, & \left(u_{1}, v_{1}\right) & =\left(u \cos \omega t_{1},-u \sin \omega t_{1}\right)=\left(\hat{u}, \sqrt{u^{2}-\hat{u}^{2}}\right), \\
T & =2 t_{1}, & \left(u_{2}, v_{2}\right) & =\left(u_{1},-v_{1}\right)=\left(\hat{u},-\sqrt{u^{2}-\hat{u}^{2}}\right) .
\end{aligned}
$$

The fundamental matrices defined by (3.15) and impact saltation matrix of (3.14) have the form

$$
X_{1}(t)=\left(\begin{array}{cc}
\cos \omega t & \sin \omega t \\
-\sin \omega t & \cos \omega t
\end{array}\right), \quad X_{2}(t)=X_{1}\left(t-t_{1}\right), \quad S=\left(\begin{array}{cc}
-1 & 0 \\
-\frac{2 u_{1}}{v_{1}} & -1
\end{array}\right),
$$

respectively. 


\section{FEČKAN - M. POSPÍŠIL}

P r o of. Due to linearity of (4.1) 0 , taken $(u, 0) \in \Sigma$ as an initial point of $\gamma^{u}(t)$ one can easily compute $\gamma_{1}^{u}(t)$ (see (3.3) $)$. Time of impact $t_{1}$ is the first intersection point of $\left\{\gamma_{1}^{u}(t) \mid t>0\right\}$ with $\Omega_{0}$ and is obtained from $h\left(\gamma_{1}^{u}\left(t_{1}\right)\right)=0$. Consequently, we get $\left(u_{1}, v_{1}\right)=\gamma_{1}^{u}\left(t_{1}\right)$ and $\left(u_{2}, v_{2}\right)=f_{2}\left(u_{1}, v_{1}\right)$ where $f_{2}(u, v)=$ $(u,-v)$ is the right-hand side of (4.2) $)_{0}$. Analogically to $\gamma_{1}^{u}(t)$ we get

$$
\gamma_{2}^{u}(t)=\left(u_{2} \cos \omega\left(t-t_{1}\right)+v_{2} \sin \omega\left(t-t_{1}\right),-u_{2} \sin \omega\left(t-t_{1}\right)+v_{2} \cos \omega\left(t-t_{1}\right)\right) .
$$

From periodicity of $\gamma^{u}(t)$ we get $T$ as a solution of equation

$$
\gamma_{2}^{u}(T)=(u, 0)
$$

or equivalently of a couple of equations

$$
\begin{array}{r}
u_{2} \cos \omega\left(T-t_{1}\right)+v_{2} \sin \omega\left(T-t_{1}\right)=u \\
-u_{2} \sin \omega\left(T-t_{1}\right)+v_{2} \cos \omega\left(T-t_{1}\right)=0 .
\end{array}
$$

We have

$$
T=t_{1}+\frac{1}{\omega} \operatorname{arccot} \frac{u_{2}}{v_{2}}=2 t_{1}
$$

Therefore using trigonometric sum identities

$$
\gamma_{2}^{u}(t)=(u \cos \omega(T-t), u \sin \omega(T-t)) .
$$

Matrices $X_{1}(t), X_{2}(t)$ and $S$ are obtained directly from their definitions since (4.1) 0 is linear.

Now we verify the basic assumptions.

Lemma 4.2. System (4.1) 0 , (4.2) $)_{0}$ satisfies conditions $\mathrm{H} 1$ ), H2) and $\mathrm{H} 3$ ).

Pr o of. From construction of $\left.\gamma^{u}(t), \mathrm{H} 1\right)$ is immediately verified. So is H2) since

$$
\operatorname{Dh}\left(u_{1}, v_{1}\right) f_{1}\left(u_{1}, v_{1}\right)=\omega v_{1}>0, \quad \operatorname{Dh}\left(u_{2}, v_{2}\right) f_{1}\left(u_{2}, v_{2}\right)=\omega v_{2}<0 .
$$

From Lemma 4.1 we get

$$
\mathrm{D}_{\xi} F(u, 0,0, \mu, \alpha)=\mathbb{I}-X_{2}(T) S X_{1}\left(t_{1}\right)=\left(\begin{array}{cc}
0 & 0 \\
\frac{2 u_{1}}{v_{1}} & 0
\end{array}\right) .
$$

Hence, it is easy to see, that $f_{1}(u, 0)=(0,-\omega u) \in Z$ for $Z$ of (3.16). Suppose that $\operatorname{dim} Z>1$. Then there exists $w \in Z$ such that $\left\langle w, f_{1}(u, 0)\right\rangle=0$, i.e., $w=(\zeta, 0)$. Next

$$
\mathrm{D}_{\xi} F(u, 0,0, \mu, \alpha) w=\left(\begin{array}{c}
0 \\
\frac{2 \zeta u_{1}}{v_{1}}
\end{array}\right) .
$$

Thus $\zeta=0$ and the verification of the last condition is completed. 
According to (3.13), by multiplying the corresponding matrices from Lemma 4.1 we derive

$$
A(t)=\left\{\begin{array}{cc}
\left(\begin{array}{cc}
\cos \omega t & -\sin \omega t \\
-\frac{2 u_{1}}{v_{1}} \cos \omega t+\sin \omega t & \frac{2 u_{1}}{v_{1}} \sin \omega t+\cos \omega t
\end{array}\right) & \text { if } t \in\left[0, t_{1}\right), \\
\left(\begin{array}{cc}
\cos \left(\omega t-2 \arccos \frac{\hat{u}}{u}\right) & -\sin \left(\omega t-2 \arccos \frac{\hat{u}}{u}\right) \\
\sin \left(\omega t-2 \arccos \frac{\hat{u}}{u}\right) & \cos \left(\omega t-2 \arccos \frac{\hat{u}}{u}\right)
\end{array}\right) & \text { if } t \in\left[t_{1}, T\right] .
\end{array}\right.
$$

Fredholm alternative yields

$$
\mathcal{R}(\mathbb{I}-A(0))^{\perp}=\mathcal{N}\left(\mathbb{I}-A^{*}(0)\right)=\mathcal{N}\left(\begin{array}{cc}
0 & \frac{2 u_{1}}{v_{1}} \\
0 & 0
\end{array}\right)=[(1,0)]
$$

thus we take $\psi=(1,0)^{*}$.

Let us consider $z(t, \varepsilon, \mu)=\sin \mu t$. It is sufficient to assume $\mu>0$ (the case $\mu<0$ is covered by parameter $\alpha \in \mathbb{R}$ ). Then Poincaré-Andronov-Melnikov function defined by (3.21) has the form

$$
\begin{aligned}
& M^{\mu}(\alpha)= \\
& =\frac{1}{2 \sqrt{l^{2}-\delta^{2}}} \int_{0}^{t_{1}}(\omega-\mu) \cos (\omega t-\mu(t+\alpha))-(\omega+\mu) \cos (\omega t+\mu(t+\alpha)) \mathrm{d} t \\
& \quad+\frac{1}{2 \sqrt{l^{2}-\delta^{2}}} \int_{t_{1}}^{T}(\omega-\mu) \cos \left(\omega t-2 \arccos \frac{\hat{u}}{u}-\mu(t+\alpha)\right) \\
& -(\omega+\mu) \cos \left(\omega t-2 \arccos \frac{\hat{u}}{u}+\mu(t+\alpha)\right) \mathrm{d} t-\frac{\sqrt{l^{2}-\delta^{2}}}{\omega l} \frac{\mu v_{1}}{u} \cos \mu\left(t_{1}+\alpha\right) .
\end{aligned}
$$

After some algebra we get

$$
M^{\mu}(\alpha)=\frac{\nu(u) \cos \mu\left(t_{1}+\alpha\right)}{\sqrt{l^{2}-\delta^{2}}}
$$

where

$$
\nu(u)=\frac{\mu\left(l^{2}-\delta^{2}\right)}{\omega l} \sqrt{1-\left(\frac{\hat{u}}{u}\right)^{2}}-2 \sin \mu t_{1} .
$$

Function $M^{\mu}(\alpha)$ can be easily differentiated with respect to $\alpha$ and one can apply Theorem 3.5 . 
Proposition 4.3. Let $0<\omega, 0<\mu$ and $k \in \mathbb{N}$ be such that $k \omega<\mu<2 k \omega$. Then for each $r \in\{0,1, \ldots, 2 k-1\}$, there exists a unique $2 k \pi / \mu$-periodic solution $x_{k, r, \varepsilon}(t)$ of system (4.1) $)_{\varepsilon}$, (4.2) $)_{\varepsilon}$ with $\varepsilon \neq 0$ sufficiently small and

$$
\alpha=\alpha_{k, r}(\varepsilon)=\frac{\pi(2 r+1)}{2 \mu}+O(\varepsilon)
$$

such that

$$
\left|x_{k, r, \varepsilon}(t)-\gamma^{u}(t-\alpha)\right|=O(\varepsilon)
$$
for any $t \in \mathbb{R}$ and $u=u(k)=\frac{\hat{u}}{\cos \frac{k \omega \pi}{\mu}}$. So there are at least $2 \sum_{k \in\left(\frac{\mu}{2 \omega}, \frac{\mu}{\omega}\right) \cap \mathbb{N}} k$ different
impact periodic solutions.

Proof. Since the forcing $\sin \mu t$ has periods $2 k \pi / \mu, k \in \mathbb{Z}$, we need the period matching condition $T=2 k \pi / \mu$ for some $k \in \mathbb{N}$. This gives

$$
2 k \pi / \mu=T=2 t_{1}=2 \frac{1}{\omega} \arccos \frac{\hat{u}}{u} .
$$

Since $\arccos \frac{\hat{u}}{u} \in(\pi / 2, \pi)$ we get the assumption $k \omega<\mu<2 k \omega$. Then

$$
u=u(k)=\frac{\hat{u}}{\cos \frac{k \omega \pi}{\mu}}<-\hat{u} .
$$

Hence

$$
\nu(u(k))=\frac{\mu\left(l^{2}-\delta^{2}\right)}{\omega l} \sin \frac{k \omega \pi}{\mu}>0 .
$$

So clearly, $M^{\mu}\left(\alpha_{0}\right)=0$ if and only if

$$
\alpha_{0}=\frac{\pi(2 s+1)}{2 \mu}-t_{1}=\frac{\pi(2(s-k)+1)}{2 \mu}
$$

for $s \in \mathbb{Z}$. In the period interval $[0, T]=[0,2 k \pi / \mu]$, we have $2 k$ different

$$
\alpha_{0} \in\left\{\frac{(2 r+1) \pi}{2 \mu} \mid r \in\{0,1, \ldots, 2 k-1\}\right\} .
$$

Obviously, each $\alpha_{0}$ is a simple root of $M^{\mu}(\alpha)$. The rest follows from Theorem 3.5

\section{Concluding remarks}

Direct combination of methods of this paper with our previous ones [12 14] can yield to further results on persistence of impact solutions of (2.1) and (2.2) such as their stability, instability or hyperbolicity. Bifurcation from degenerate impact periodic orbit, i.e., when H3) fails, can be also studied like in [14. Moreover, persistence from a family of impact periodic solutions, i.e., when there is a family of periodic impact orbits in assumption H1), can be investigated 


\section{PERSISTENCE OF PERIODIC ORBITS IN PERIODICALLY FORCED IMPACT SYSTEMS}

analogically as in [13. Since all these extensions and generalizations can be done straightforwardly, we do not go into details in this paper. Of course, the appropriated derivations are not elementary.

Acknowledgement. The authors thank referee for useful comments and suggestions.

\section{REFERENCES}

[1] AFSHARNEZHAD, Z.-KARIMI AMALEH, M.: Continuation of the periodic orbits for the differential equation with discontinuous right hand side, J. Dynam. Differential Equations 23 (2011), 71-92.

[2] AKHMET, M. U.-ARUGASLAN, D.: Bifurcation of a non-smooth planar limit cycle from a vertex, Nonlinear Anal. 71 (2009), 2723-2733.

[3] AWREJCEWICZ, J.-HOLICKE, M. M.: Smooth and Nonsmooth High Dimensional Chaos and the Melnikov-Type Methods, World Scientific Publishing Company, Singapore, 2007.

[4] DI BERNARDO, M.-BUDD, C. J.-CHAMPNEYS, A. R.-KOWALCZYK, P.: Piecewise-smooth Dynamical Systems: Theory and Applications. Appl. Math. Sci. 163, Springer-Verlag, London, 2008.

[5] BROGLIATO, B.: Nonsmooth Impact Mechanics. Lecture Notes in Control and Inform. Sci. 220, Springer, Berlin, 1996.

[6] CHICONE, C.: Ordinary Differential Equations with Applications. Texts Appl. Math. 34, Springer, New York, 2006.

[7] CHILlingWORTH, D. R. J.: Discontinuous geometry for an impact oscillator, Dyn. Syst. 17 (2002), 389-420.

[8] CHOW, S. N.-HALE, J. K.: Methods of Bifurcation Theory, Springer-Verlag, New York, 1982.

[9] DeIMLING, K.: Nonlinear Functional Analysis, Springer-Verlag, Berlin, 1985.

[10] DU, Z.-ZHANG, W.: Melnikov method for homoclinic bifurcation in nonlinear impact oscillators, Comput. Math. Appl. 50 (2005), 445-458.

[11] FARKAS, M.: Periodic Motions, Springer-Verlag, New York, 1994.

[12] FEČKAN, M.-POSPÍŠIL, M.: On the bifurcation of periodic orbits in discontinuous systems, Commun. Math. Anal. 8 (2010), 87-108.

[13] FEČKAN, M.-POSPÍŠIL, M.: Bifurcation from family of periodic orbits in discontinuous autonomous systems, Differential Equations Dynam. Systems 20 (2012), 207-234.

[14] FEČKAN, M.-POSPÍŠIL, M.: Bifurcation from single periodic orbit in discontinuous autonomous systems, Appl. Anal. 92 (2013), 1085-1100.

[15] FIDLIN, A.: Nonlinear Oscillations in Mechanical Engineering, Springer, Berlin, 2006.

[16] HARTMAN, P.: Ordinary Differential Equations, John Wiley \& Sons, Inc., New York, 1964.

[17] KOVALEVA, A.: The Melnikov criterion of instability for random rocking dynamics of a rigid block with an attached secondary structure, Nonlinear Anal. Real World Appl. 11 (2010), 472-479. 


\section{FEČKAN - M. POSPÍŠIL}

[18] KUKUČKA, P.: Jumps of the fundamental solution matrix in discontinuous systems and applications, Nonlinear Anal. 66 (2007), 2529-2546.

[19] KUNZE, M.: Non-smooth Dynamical Systems, Lecture Notes in Math. 1744, Springer, Berlin-New York, 2000.

[20] LEINE, R. I.-NIJMEIJER, H.: Dynamics and Bifurcations of Non-smooth Mechanical Systems, Lect. Notes Appl. Comput. Mech. 18, Springer-Verlag, Berlin, 2004.

[21] LENCI, S.-REGA, G.: Heteroclinic bifurcations and optimal control in the nonlinear rocking dynamics of generic and slender rigid blocks, Internat. J. Bifur. Chaos Appl. Sci. Engrg. 15 (2005), 1901-1918.

[22] MAKARENKOV, O.-VERHULST, O.: Bifurcation of asymptotically stable periodic solutions in nearly impact oscillators. Preprint [arXiv:0909.4354v1].

[23] XU, W.-FENG, J.-RONG, H.: Melnikov's method for a general nonlinear vibro-impact oscillator, Nonlinear Anal. 71 (2009), 418-426.

Received 6. 9. 2011

Accepted 8. 1. 2012

\author{
* Department of Mathematical Analysis \\ and Numerical Mathematics \\ Comenius University \\ Mlynská dolina \\ SK-842 48 Bratislava \\ SLOVAKIA \\ Mathematical Institute \\ Slovak Academy of Sciences \\ Štefánikova 49 \\ SK-814 73 Bratislava \\ SLOVAKIA
}

E-mail: Michal.Feckan@fmph.uniba.sk

** Centre for Research and

Utilization of Renewable Energy

Faculty of Electrical Engineering

and Communication

Brno University of Technology

Technická 3058/10

CZ-616 OO Brno CZECH REPUBLIC

E-mail: pospisilm@feec.vutbr.cz 